\title{
ORIGINAL RESEARCH \\ Effect of Antiplatelet Therapy on Thromboembolic Complications of Elective Coil Embolization of Cerebral Aneurysms
}

\author{
N.K. Yamada \\ D.T. Cross III \\ T.K. Pilgram \\ C.J. Moran \\ C.P. Derdeyn \\ R.G. Dacey, Jr
}

\begin{abstract}
BACKGROUND AND PURPOSE: Thromboembolic events are the most common complications of elective coil embolization of cerebral aneurysms. Administration of oral clopidogrel and/or aspirin could lower the thromboembolic complication rate.
\end{abstract}

MATERIALS AND METHODS: Records over a 10-year period were reviewed in a retrospective cohort study. For 369 consecutive elective coil embolization procedures, 25 patients received no antiplatelet drugs, 86 received antiplatelet drugs only after embolization, and 258 received antiplatelet drugs before and after embolization.

RESULTS: Symptomatic thromboembolic complications (transient ischemic attack or stroke within 60 days) occurred in $4(16 \%)$ of 25 when no antiplatelet drugs were given, in $2(2.3 \%)$ of 86 when antiplatelet drugs were administered only after embolization, and in 5 (1.9\%) of 258 when antiplatelet drugs were administered before and after embolization. The lower symptomatic thromboembolic complication rate in the patients who received any antiplatelet therapy was statistically significant $(P=$ .004). Clots were visible intraprocedurally in 5 (4.5\%) of 111 when no antiplatelet drugs were administered before procedures and in $4(1.6 \%)$ of 258 when they were ( $P$ value not significant). None of the 9 was symptomatic postprocedurally, but 7 were lysed or mechanically disrupted. Extracerebral hemorrhagic complications occurred in $0(0 \%)$ of 25 when no antiplatelet drugs were given and in 11 (3.2\%) of 344 when they were ( $P$ value not significant).

CONCLUSION: Oral clopidogrel and/or aspirin significantly lowered the symptomatic thromboembolic complication rate of elective coil embolization of unruptured cerebral aneurysms. There were trends toward a lower rate of intraprocedural clot formation in patients given antiplatelet drugs before procedures and a higher hemorrhagic complication rate in patients given antiplatelet drugs. Benefits of antiplatelet therapy appear to outweigh risks.

$\mathbf{M}$ ost complications resulting from transarterial coil embolization of cerebral aneurysms are thromboembolic. ${ }^{1}$ Antiplatelet drugs, including aspirin and clopidogrel (Plavix), are widely prescribed for elective endovascular treatment of cerebral aneurysms in an effort to lower the thromboembolic complication rate, but data reported to support that practice are currently limited to 1 study in which intravenous aspirin was used. ${ }^{2}$ We sought to determine whether the administration of oral antiplatelet agents lowered the thromboembolic complication rate or increased the hemorrhagic complication rate resulting from elective coil embolization of cerebral aneurysms through a retrospective review of clinical data.

\section{Patients and Methods}

\section{Patient Population}

This retrospective cohort study was approved by the human studies committee. Endovascular treatment procedure logs and departmental patient listings were reviewed to identify all of the patients who underwent elective coil embolization of cerebral aneurysms at our

Received November 24, 2006; accepted after revision March 14, 2007.

From the Departments of Radiology (N.K.Y., D.T.C., T.K.P., C.J.M., C.P.D.) and Neurology and Neurological Surgery (D.T.C., C.J.M., C.P.D., R.G.D.), Washington University School of Medicine, St. Louis, Mo.

This work was supported by the Ruth L. Kirschstein National Research Service Award, National Institutes of Health (to N.K.Y.).

Please address correspondence to DeWitte T. Cross III, Washington University School of Medicine, Campus Box 8131, 510 S. Kingshighway Blvd, St. Louis, MO 63110; e-mail: CrossDe@wustl.edu

DOI 10.3174/ajnr.A0641 institution between December 1, 1995, and December 31, 2005. Patients excluded were those who presented with acutely ruptured aneurysms (ie, subarachnoid hemorrhage or intraventricular hemorrhage less than 30 days before embolization), those who received warfarin (Coumadin) at any time in the course of endovascular treatment, those who received antiplatelet drugs only before and not after embolization, and those for whom records were incomplete or not available.

\section{Drug Regimens}

All of the patients were systemically anticoagulated with intravenous heparin during coil embolization procedures with initial boluses of 70-100 $\mathrm{U} / \mathrm{kg}$ followed by continuous infusions of $7-10 \mathrm{U} / \mathrm{kg}$ per hour. Activated clotting times on heparin were monitored and maintained at least twice baseline, usually at or near 300 seconds, by administration of additional boluses or by adjusting infusion rates, as needed. Heparin was generally discontinued at the conclusion of the procedures. Patients given antiplatelet drugs always received oral aspirin, 81 or $325 \mathrm{mg}$ daily, unless contraindicated by an aspirin allergy. Since 1999, when clopidogrel became available, many patients also received clopidogrel for 3-5 days before their procedures. In a minority of cases, clopidogrel was given in loading doses of 300-450 mg immediately before procedures. Patients treated after procedures were generally given aspirin permanently and clopidogrel for 30-60 days.

\section{Chart Review}

Hospital and clinic records and departmental quality assurance (QA) logs were reviewed, and the following information was recorded in a spreadsheet: date of birth; date of procedure; date of previous or sub- 
Table 1: Treatment group demographics

\begin{tabular}{|c|c|c|c|c|c|}
\hline Variable & $\begin{array}{c}\text { Antiplatelet Drugs: } \\
\text { Before and After }\end{array}$ & $\begin{array}{c}\text { Antiplatelet } \\
\text { Drugs: After Only }\end{array}$ & $\begin{array}{l}\text { No Antiplatelet } \\
\text { Drugs }\end{array}$ & $P$ & Statistical Test \\
\hline Women & $188 / 258(72.9)$ & $69 / 86(80.2)$ & $20 / 25(80.0)$ & .3285 & $x^{2}(2 \times 3)$ \\
\hline Men & 70/258 (27.1) & $17 / 86(19.8)$ & $5 / 25(20.0)$ & .3285 & $x^{2}(2 \times 3)$ \\
\hline Age, y & 55.68 & 53.45 & 57.80 & .1922 & One-way ANOVA \\
\hline Diameter of first framing coil, mm & 6.3 & 7.0 & 7.3 & .1834 & One-way ANOVA \\
\hline Prolapsed coil & $30 / 258(11.6)$ & 8/86 (9.3) & $3 / 25(12.0)$ & .7840 & $x^{2}(2 \times 3)$ \\
\hline Coated coils & $64 / 258(24.8)$ & 10/86 (11.6) & $1 / 25(4.0)$ & $.0014^{*}$ & $x^{2}(2 \times 3)$ \\
\hline Balloon & $32 / 258(12.4)$ & $8 / 86(9.3)$ & $1 / 25(4.0)$ & .3098 & $x^{2}(2 \times 3)$ \\
\hline Stent (total) & $52 / 258(20.2)$ & $6 / 86(7.0)$ & $0 / 25(0.0)$ & $.0001^{*}$ & $x^{2}(2 \times 3)$ \\
\hline
\end{tabular}

Note:-Raw values are reported in each cell. Percentage of the population is in parentheses. ANOVA indicates analysis of variance.

* denotes statistically significant $P$ value.

Table 2: Frequency of thromboembolic complications by treatment category

\begin{tabular}{lcccc}
\hline & \multicolumn{2}{c}{ Symptomatic Thromboembolic Complication } \\
\cline { 2 - 5 } Treatment Category & $\begin{array}{c}\text { Postprocedure TIA } \\
(\leq 24 \mathrm{~h})\end{array}$ & $\begin{array}{c}\text { Postprocedure Ischemic } \\
\text { Stroke }(\leq 24 \mathrm{~h})\end{array}$ & $\begin{array}{c}\text { TIA Between } 1 \\
\text { and } 60 \text { Days }\end{array}$ & $\begin{array}{c}\text { Ischemic Stroke Between } \\
1 \text { and } 60 \text { Days }\end{array}$ \\
\hline Antiplatelet therapy (before and after) & 1 & 0 & $3^{*}$ & $1 \dagger$ \\
Antiplatelet therapy (after only) & 0 & 0 & $2 \ddagger$ & 0 \\
No drugs & 2 & $1 \S$ & 0 & $2 \S$ \\
\hline
\end{tabular}

Note:-TIA indicates transient ischemic attack.

* One each at 3,39 , and 60 days postprocedure.

† At 50 days postprocedure.

$\ddagger$ One each at 34 and 38 days postprocedure.

$\S$ One patient suffered 2 ischemic strokes, 1 within 24 hours and 1 at 6 days postprocedure

sequent surgery for the same or other aneurysms; antiplatelet therapy (ie, clopidogrel and/or aspirin) administered before and/or after endovascular treatment; occurrence of postprocedure transient ischemic attack (TIA) or stroke within 24 hours; occurrence of TIA or stroke within 60 days and date; hemorrhage within 60 days; and results of follow-up conventional angiography or MR angiography. An ischemic clinical event was defined as a TIA if symptoms were less than 24 hours in duration or as a stroke if symptoms lasted more than 24 hours. Every patient recovered postprocedurally in the neurologyneurosurgery intensive care unit, where neurologic examinations were performed by neurologists and neurosurgeons who were not involved in the interventional procedures. Neurologic examinations performed after hospital discharge were performed by neurosurgeons or interventional neuroradiologists who, in some cases, may have been involved in the interventional procedures.

\section{Procedure Reports}

The procedure reports of endovascular treatment were reviewed for the following: aneurysm dome size, neck size, and location; whether the aneurysm was ruptured or unruptured; type of coils used (ie, bare platinum or coated); total number of coils placed; diameter of the first framing coil; degree of aneurysm obliteration and presence of a residual aneurysm neck after coil embolization; endovascular technique modification (ie, use of balloon remodeling and/or stent deployment); presence of coil(s) protruding into parent vessel; occurrence of intra-arterial thrombus; and occurrence of periprocedural TIA, stroke, or hemorrhage.

\section{Statistical Analysis}

In patients with more than 1 aneurysm, each aneurysm treatment was considered as a separate source for thromboembolic events. Aneurysms requiring retreatment because of coil compaction were considered new aneurysms from the time of retreatment forward, and the follow-up period for the original treatment was concluded.

For statistical analysis, aneurysm size was defined by the diameter of the first framing coil detached within the aneurysm, because framing coils detached were consistently documented in procedure reports, but measurements of aneurysm diameters were not. The relationship of clinical, anatomic, and pharmacologic factors to either periprocedural or postprocedural ischemic and hemorrhagic events was investigated. Ischemic and hemorrhagic events were investigated separately because different mechanisms are involved. Age, sex, use of coated coils, coils prolapsed into the parent artery, endovascular technique modifications such as balloon remodeling or stent-assisted coiling, and the administration of antiplatelet drugs before and/or after endovascular treatment were analyzed as dichotomous independent variables. Age and diameter of the first framing coil were analyzed as continuous independent variables. The association of these factors with either periprocedural or postprocedural ischemic events was tested in a univariate analysis using $\mathrm{X}^{2}$ or Fisher exact tests for dichotomous variables and logistic regression for continuous variables. Statistical significance was accepted at a $P<.05$.

\section{Results}

Review of procedure notes, radiographic reports, QA logs, inpatient records, and clinic records revealed 684 consecutive coil embolizations. Excluded from analysis were 296 embolizations performed for subarachnoid or intraventricular hemorrhage, 15 for which Coumadin was given, and 4 for which antiplatelet drugs were given before procedures and records were incomplete after procedures. The 369 embolizations remaining for analysis represent 333 patients with 1 elective embolization and 18 patients with 2 . Of these, 25 embolizations were performed with no antiplatelet drugs given, 86 with antiplatelet drugs given only after coil embolization procedures, and 258 with antiplatelet drugs given before and after the procedures (Table 1).

Symptomatic thromboembolic complications were identified after 11 embolization procedures (Table 2). Five of the 11 were among the 258 embolizations where antiplatelet therapy 


\begin{tabular}{|c|c|c|c|}
\hline \multicolumn{4}{|c|}{$\begin{array}{l}\text { Table 3: Treatment category versus thromboembolic complication } \\
\text { rate }\end{array}$} \\
\hline \multirow{2}{*}{$\begin{array}{l}\text { Treatment } \\
\text { Category }\end{array}$} & \multicolumn{3}{|c|}{ Symptomatic Thromboembolic Complication } \\
\hline & No, $n($ Row $\%)$ & Yes, $n$ (Row \%) & Total $N$ \\
\hline $\begin{array}{l}\text { Antiplatelet therapy } \\
\text { (before and after) }\end{array}$ & $253(98.1)$ & $5(1.9)$ & 258 \\
\hline $\begin{array}{l}\text { Antiplatelet therapy } \\
\text { (after only) }\end{array}$ & $84(97.7)$ & $2(2.3)$ & 86 \\
\hline No drug & $21(84.0)$ & $4(16.0)$ & 25 \\
\hline Total $N$ & 358 & 11 & 369 \\
\hline
\end{tabular}

Note: $-X^{2}$ indicates likelihood ratio; $P=.0133$. The percentage of patients in the "before and after" group (1.9\%) who had thromboembolic complications is very comparable with the percentage of patients in the "after only" group $(2.3 \%)$.

\begin{tabular}{l}
$\begin{array}{l}\text { Table 4: Binary treatment category versus thromboembolic } \\
\text { complication rate }\end{array}$ \\
\hline \begin{tabular}{lccc} 
Binary Treatment & Symptomatic Thromboembolic Complication \\
\cline { 2 - 4 } Category & No, $n$ (Row \%) & Yes, $n$ (Row \%) & Total $N$ \\
\hline Antiplatelet therapy & $337(98.0)$ & $7(2.0)$ & 344 \\
No drug & $21(84.0)$ & $4(16.0)$ & 25 \\
Total $N$ & 358 & 11 & 369 \\
\hline
\end{tabular}
\end{tabular}

Note:-Fisher exact test indicates 2-tail $P=.0039$. An alternative hypothesis is that thromboembolic complication rate is different across treatment categories.

was given before and after endovascular treatment. In those 5, 1 TIA occurred within 24 hours of the procedure, 1 TIA occurred 3 days postprocedure, 2 TIAs occurred between 30 and 60 days postprocedure, and 1 cerebral vascular accident (CVA) occurred 50 days postprocedure. Two of the 11 were among the 86 embolizations where antiplatelet therapy was given only after treatment. In both, TIAs occurred between 30 and 60 days postprocedure. Four of the 11 were among the 25 embolizations where no antiplatelet therapy was given. In those 4 embolizations, 2 patients had TIAs occurring within 24 hours of the procedure, 1 patient had a CVA occurring 2 days postprocedure, and 1 patient had 2 CVAs, 1 within 24 hours and one 6 days postprocedure. No patient who had a thromboembolic complication had a stent placed or had coated coils placed. Two patients who were treated with antiplatelet drugs and 1 patient who was not treated with antiplatelet drugs had thromboembolic complications after balloon remodeling.

As shown in Table 3, cases where antiplatelet therapy was administered before and after treatment were not significantly different from cases where antiplatelet therapy was administered only after treatment. Thus, these 2 groups were combined, and the thromboembolic complication rate in cases with no antiplatelet therapy at all (4 of 25 [16\%]) was compared with the rate in those with antiplatelet therapy at any point in their treatment (7 of 344 [2\%]). A Fisher exact test revealed a statistically significant decrease in thromboembolic complications in patients who received antiplatelet therapy (Table 4; $P=.0039$ ). With TIAs removed and CVAs only analyzed as symptomatic thromboembolic complications, the patterns remained statistically significant by using the Fisher exact test $(P=.01)$, but cell counts are small.

Age, sex, diameter of the first framing coil (as a marker for aneurysm diameter), presence of prolapsed coils, use of coated coils, and endovascular technique modifications (balloon remodeling and stent placement) were evaluated to determine whether there were other variables associated with symptomatic thromboembolic events. None of these was significantly

\begin{tabular}{|c|c|c|c|}
\hline \multicolumn{4}{|c|}{$\begin{array}{l}\text { Table 5: Binary treatment category versus hemorrhagic } \\
\text { complication rate }\end{array}$} \\
\hline \multirow{2}{*}{$\begin{array}{l}\text { Binary Treatment } \\
\text { Category }\end{array}$} & \multicolumn{3}{|c|}{ Hemorrhagic Complication } \\
\hline & No, $n$ (Row \%) & Yes, $n($ Row \%) & Total $N$ \\
\hline Antiplatelet therapy & $332(96.8)$ & $11(3.2)$ & 343 \\
\hline No drug & $24(100.0)$ & $0(0.0)$ & 24 \\
\hline Total N & 356 & 11 & 367 \\
\hline
\end{tabular}

Note:-Fisher exact test indicates 2-tail $P=1.000$. An alternative hypothesis is that hemorrhagic complication rate is different across treatment categories.

associated with the symptomatic thromboembolic complication rate $(P \geq .1)$. These same variables were all potentially confounding factors in this study; however, bivariate analysis revealed that only coated coils $(P=.001)$ and stent placement $(P=.0001)$ had statistically significant relationships with treatment category.

Intra-arterial thrombus was visible angiographically during embolization procedures but not associated with postprocedural ischemic symptoms in $4(1.6 \%)$ of 258 pretreated with antiplatelet drugs and in 5 (4.5\%) of 111 not pretreated with antiplatelet drugs. This was not a statistically significant difference $(P=.14)$. Of the 9 cases in which thrombi or emboli were seen intraprocedurally, intra-arterial fibrinolytic drugs (tissue plasminogen activator, urokinase, or retevase) were given to 6 , mechanical disruption of a clot with a microwire was performed in 1, additional heparin was given in 1, and no additional treatment was given in 1 . There were no hemorrhagic complications in this group of patients.

Thirteen hemorrhagic complications were identified. One from the group receiving antiplatelet drugs and 1 from the group not receiving antiplatelet drugs were excluded, because the hemorrhages were precipitated by perforation of an arterial wall during coiling procedures, a complication not related to drug therapy. Both of those patients survived without permanent neurologic deficits. The remaining 11 cases all involved patients who had received antiplatelet therapy. The most common hemorrhagic complication was retroperitoneal hematoma (5 cases), with inguinal hematoma or bleeding at the puncture site ( 3 cases) and nasal or oral bleeding related to intubation (3 cases) as less frequent complications. The relationship between drug treatment category and hemorrhagic complication rate was analyzed through bivariate analysis and failed to reach statistical significance (Table 5; $P>$.999).

\section{Discussion}

Although the overall complication rate for elective endovascular treatment of cerebral aneurysms is relatively low, thromboembolic complications remain the most frequent (Fig 1). The combined rate of TIA and nonhemorrhagic stroke in clinical data submitted for the 1995 US Food and Drug Administration approval of the Guglielmi detachable coil (GDC), the first detachable coil introduced for aneurysm embolization, was $5.6 \%$, a figure that reflected symptomatic thromboembolic complications resulting from treatment of 770 aneurysms (52\% of which were elective) in 735 patients (Lucie Thibault, Target Therapeutics, Fremont, Calif, written communication, GDC US Clinical Study Summary, 1996). A published subset of those cases, basilar tip aneurysms treated with GDCs, documented a 5\% thromboembolic complication rate for elective cases. ${ }^{3}$ After detachable coils were used clinically 

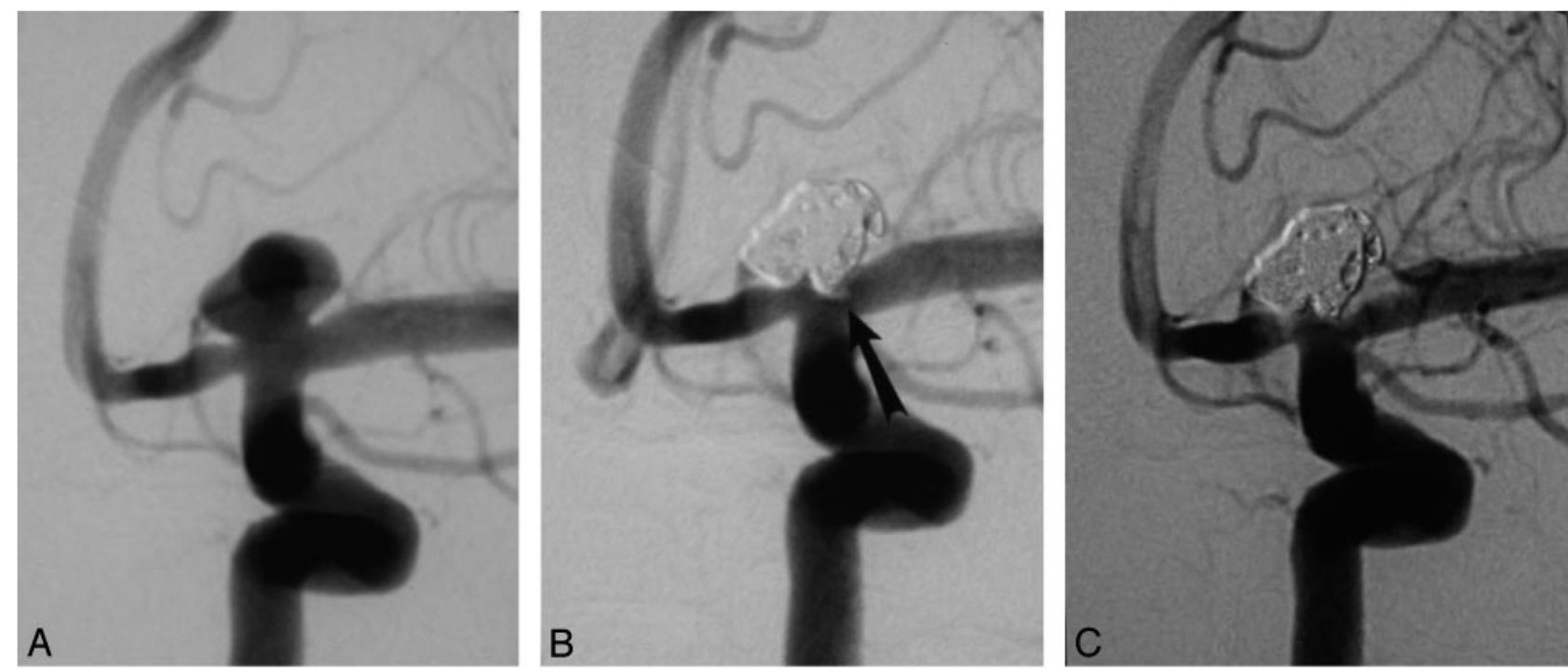

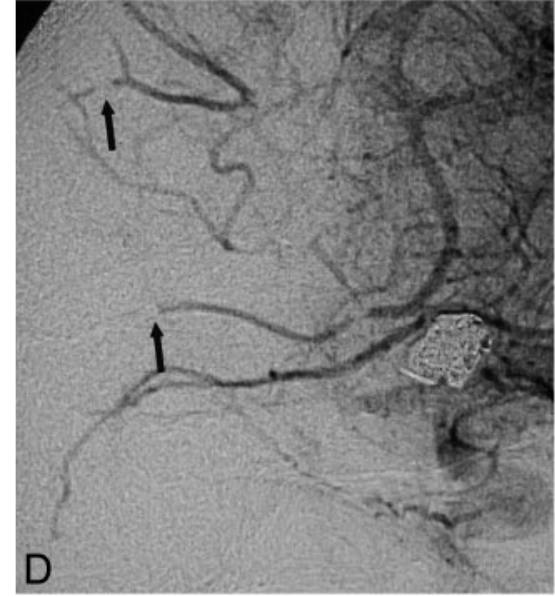

for cerebral aneurysm treatment for approximately 4 years in the United States, the rate of ischemic complications reported in a meta-analysis of 1265 ruptured and unruptured cases in 1999 was $8.5 \%{ }^{4}$ Subsequent reports have cited both higher ${ }^{5-7}$ and lower ${ }^{1}$ thromboembolic complication rates, with the higher rates in part attributed to treatment of previously untreated aneurysms with greater diameters and wider necks, made possible through modified embolization techniques and the lower rates attributed to institution of antiplatelet therapy. ${ }^{8}$

Elective coil embolization of cerebral aneurysms was consistently performed under systemic heparin anticoagulation at our institution from the beginning, but antiplatelet therapy for elective aneurysm coiling was inconsistently used in our clinical practice from 1995 through 1998. Data from percutaneous coronary intervention studies showed that antiplatelet therapy in addition to heparin anticoagulation provided improved protection from thromboembolic complications. ${ }^{9,10}$ Based on benefits observed for coronary interventions with implantable devices, antiplatelet drugs (clopidogrel and/or aspirin) were more consistently administered by us for elective endovascular cerebral aneurysm treatment from 1999 to the present. Internal QA data suggested that the thromboembolic complication rate associated with cerebral aneurysm coiling fell with the institution of antiplatelet therapy, leading to the conduct of this investigation.
In this retrospective study, antiplatelet therapy was shown to lower the thromboembolic complication rate from $16 \%$ (4 of $25 ; 95 \%$ confidence limits, $5.2 \%-36.9 \%$ ) in the no-drug cohort to $2 \%$ ( 7 of $344 ; 95 \%$ confidence limits, $0.9 \%-4.3 \%$ ) in patients who received antiplatelet therapy either after or before and after endovascular treatment. The increased use of coated coils and endovascular technique modifications (eg, stent-assisted coiling) in patients who received antiplatelet therapy before and after treatment reflects the chronologic development of these technologies. These 2 techniques are more recent developments in endovascular therapy, and their greater use parallels the more common use of antiplatelet therapy both before and after treatment.

There was no statistical difference between the symptomatic thromboembolic complication rates of elective cerebral aneurysm coiling in the patients treated with antiplatelet drugs before and after the procedure and the patients treated with antiplatelet drugs only after the procedure; however, there was a trend toward intra-arterial thrombus formation in patients who did not receive antiplatelet drugs before procedures ( $4.5 \%$ versus $1.6 \%$ ), and most of the patients who developed a clot intraprocedurally were given intra-arterial fibrinolytic drugs. Those patients in whom thrombi or emboli developed during procedures would probably not all have been asymptomatic postprocedurally without the added interventions. Although not statistically significant in this study, the development of thrombus intraprocedurally more commonly in patients not pretreated with antiplatelet drugs, together with the statistically nonsignificant difference in the hemorrhagic complication rate, probably merits further prospective investigation in a greater number of subjects. It may be that limiting antiplatelet therapy to the postprocedure period would limit hemorrhagic complications but would predispose patients to intra-arterial intraprocedural thrombus formation requiring further intervention.

The presence of prolapsed coils may serve as a predictor for subsequent ischemic events. ${ }^{11-13}$ Coils that have prolapsed into the parent artery may serve as an additional site for plate- 
let aggregation, which can then lead to thrombosis. An association between prolapsed coils and increased risk for thromboembolic complications and an association reported previously between increased aneurysm diameter and increased risk for thromboembolic complications ${ }^{11}$ failed to achieve statistical significance in this study.

Interestingly, there were no patients treated with coated coils or stents who experienced symptomatic thromboembolic complications. Nevertheless, there was no significant relationship between either variable and the complication rate.

The numbers of patients were insufficient to further subdivide cohort groups into those receiving aspirin alone, clopidogrel alone, and aspirin and clopidogrel combined, without losing statistical power. Further prospective evaluation may determine whether 2 drugs are synergistic in preventing symptomatic thromboembolic complications without increasing hemorrhagic complications.

There are several limitations of this study that require acknowledgment. First, the sample size of the no-drug cohort was relatively small. Second, the frequency of thromboembolic complications was low. Third, this was a retrospective study. It is possible that some medical records were incomplete, leading to an inaccurate assignment of patients into the antiplatelet drug treatment categories. There may have been some biases in patient assignments to the different treatment schedules. For example, any patient in whom stent placement was anticipated to facilitate aneurysm coiling was pretreated with antiplatelet drugs, and treatment with antiplatelet drugs was more common later in the series. None of these limitations affected our ability to find an association between antiplatelet therapy and decreased incidence of thromboembolic complications.

\section{Conclusion}

Oral clopidogrel and/or aspirin significantly lowered the symptomatic thromboembolic complication rate of elective coil embolization of unruptured cerebral aneurysms. There were trends toward a lower rate of intraprocedural clot formation in patients given antiplatelet drugs before procedures and toward a higher hemorrhagic complication rate in patients given antiplatelet drugs. The benefits of antiplatelet therapy instituted before and continued after these procedures appear to outweigh the risks.

\section{Acknowledgments}

We thank Tracy Dobbie for assistance with data, chart, and report retrieval vital for the conduct of this study.

\section{References}

1. Lantera L, Tredici G, Dimitrov B, et al. Treatment of unruptured cerebral aneurysms by embolization with Guglielmi detachable coils: case fatality, morbidity, and effectiveness in preventing bleeding - a systematic review of the literature. Neurosurgery 2004;55:767-78

2. Thorsten R, Jan-Hendrik B, Kucinski T, et al. Intravenous administration of acetylsalicylic acid during endovascular treatment of cerebral aneurysms reduces the rate of thromboembolic events. Stroke 2006;37:1816-21

3. Eskridge JM, Song JK. Endovascular embolization of 150 basilar tip aneurysms with Guglielmi detachable coils: results of the Food and Drug Administration multicenter clinical trial. J Neurosurg 1998;89:81-86

4. Brilstra EH, Rinkel GJE, van der Graaf Y, et al. Treatment of intracranial aneurysms with coils: a systematic review. Stroke 1999;30:470-76

5. Albayram S, Selcuk H, Kara B, et al. Thromboembolic events associated with balloon-assisted coil embolization: evaluation with diffusion-weighted MR imaging. AJNR Am J Neuroradiol 2004;25:1768-77

6. Pelz DM, Lownie SP, Fox AJ. Thromboembolic events associated with the treatment of cerebral aneurysms with Guglielmi detachable coils. AJNR Am J Neuroradiol 1998;19:1541-47

7. Pouratian N, Oskouian RJ, Jensen ME, et al. Endovascular management of unruptured intracranial aneurysms. J Neurol Neurosurg Psychiatry 2006;77:572-78

8. Henkes H, Fischer S, Weber W, et al. Endovascular coil occlusion of 1811 intracranial aneurysms: early angiographic and clinical results. Neurosurgery 2004;54:268-85

9. CAPRIE Steering Committee. A randomised, blinded, trial of clopidogrel versus aspirin in patients at risk of ischaemic events (CAPRIE). Lancet 1996;348:1329-39

10. Steinhubl SR, Berger PB, Mann JT 3rd, et al. Early and sustained dual ora antiplatelet therapy following percutaneous coronary intervention: a randomized controlled trial. JAMA 2002;288:2411-20

11. Derdeyn CP, Cross DT, Moran CJ, et al. Postprocedure ischemic events after treatment of intracranial aneurysms with Guglielmi detachable coils. J Neurosurg 2002;96:837-43

12. Klotzsch C, Nahser HC, Henkes H, et al. Detection of microemboli distal to cerebral aneurysms before and after therapeutic embolization. AJNR Am J Neuroradiol 1998;19:1315-18

13. Malisch TW, Guglielmi G, Vinuela F, et al. Intracranial aneurysms treated with the Guglielmi detachable coil: midterm clinical results in a consecutive series of 100 patients. J Neurosurg 1997;87:176-83 